\title{
Estudios sobre procesos educativos en organizaciones y movimientos sociales
}

\author{
Studies on educational processes \\ in organizations and social movements \\ Estudos sobre processos educativos em \\ organizações e movimentos sociais
}

Disney Barragán Cordero ${ }^{1}$ Alfonso Torres Carrillo²

\section{Resumen}

Los estudios sobre la educación en movimientos sociales en América Latina, se remontan a la segunda mitad de la década de 1980. Sus temas de interés, así como sus claves de comprensión han variado, de la mano de las teorías socioeducativas, pasando de las posiciones más reproductivistas, a unas que acentúan su carácter de resistencia, hasta las actuales, que ven la educación como estrategia de formación de subjetividades emancipadoras e imaginarios instituyentes, que recuperan el sujeto y sus capacidades en la transformación social. El objetivo de este artículo de revisión es hacer un balance del conocimiento producido desde los estudios sobre la relación entre educación y movimientos sociales en torno a las temáticas tales como los sujetos, los sentidos, los contenidos y las estrategias de las prácticas educativas. A partir de un balance de sus aportes al campo en construcción de la pedagogía de las subjetividades emancipadoras y los sujetos sociales, planteamos algunos desafíos para este campo de investigación.

\section{Palabras clave}

prácticas educativas; pedagogía; movimientos sociales; subjetividades; sujetos sociales; formación

\section{Abstract}

Studies on education in social movements in Latin America go back to the second half of the 1980s. Their topics of interest, as well as the keys to understanding have changed together with socio-educational theories, going from the most reproductivist positions, to ones that accentuate their character of resistance, up to the current ones, that see education as a strategy for the formation of emancipatory subjectivities and instituting imaginaries that recover the subject and its capacities in social transformation. The purpose of this review paper is to take stock of the knowledge generated from studies on the relationship between education and social movements around thematic subjects such as the subjects, the senses, the contents and the strategies of educational practices. Based on the assessment of their contributions to the field of construction of the pedagogy of emancipatory subjectivities and social subjects, we pose some challenges for this field of research.

\section{Keywords}

educational practices; pedagogy; social movements; subjectivities; social subjects; training

1 Doctora en Educación. Profesora de la maestría en Estudios Sociales, Universidad Pedagógica Nacional.

Correo electrónico: dbarragan@pedagogica.edu.co

2 Doctor en Estudios Latinoamericanos. Profesor de la maestría en Estudios Sociales, Universidad Pedagógica Nacional. Correo electrónico: atorres@pedagogica.edu.co 


\section{Resumo}

Os estudos sobre educação em movimentos sociais na América Latina remontam à segunda metade da década de 1980. 0s temas de interesse, assim como as chaves para a compreensão variam, junto com as teorias socioeducativas, desde as posições mais reprodutivistas, as que ressaltam seu caráter de resistência, até às actuais, que percebem a educação como estratégia de formação de subjetividades emancipadoras e imaginários instituintes, que resgatam o sujeito e suas capacidades na transformação social. 0 objetivo deste artigo de revisão é apresentar um balanço do conhecimento produzido desde os estudos sobre a relação entre educação e movimentos sociais em torno a temáticas como os sujeitos, os sentidos, os conteúdos e as estratégias das práticas educacionais. A partir de um balanço de suas contribuições para o campo em construção da pedagogia das subjetividades emancipadoras e os sujeitos sociais, propomos alguns desafios para este campo de pesquisa.

\section{Palavras-chave}

práticas educacionais; pedagogia; movimentos sociais; subjetividades; sujeitos sociais; formação

\section{Artículo recibido el 3 de octubre de 2017 y aprobado el 10 de enero de 2018}

\section{Introducción}

Este artículo es un balance de la producción bibliográfica y hemerográfica en torno a la problemática de la educación en los movimientos sociales (MS) en América Latina; por ello, en un primer lugar, se describen las principales tendencias encontradas en los documentos analizados entre 1986 y 2017 , periodo en el que se viene configurando como campo de investigación social y pedagógico. En segundo lugar, se hace un balance del conocimiento producido en torno a las temáticas más recurrentes: formación de militantes como sentido de las propuestas; organización o el movimiento como sujeto educativo; pedagogías de los movimientos como aporte epistémico, pedagógico y político, y formación de subjetividades y sujetos sociales. Desde este balance, se plantean algunos desafíos para este campo de investigación.

\section{Los estudios sobre educación en movimientos sociales: una mirada de conjunto}

El corpus para este estado del arte fueron 100 documentos, entre artículos, libros y trabajos de grado, tres de los cuales son estados del arte, centrados en movimientos campesinos y rurales (Souza, 2007; Palumbo, 2016) o en un país, Argentina (Baraldo, 2010). Sesenta y cinco documentos corresponden a artículos en revistas universitarias; 24 a libros; 5 a tesis de posgrado, y 6 a artículos en páginas web. Del total de los documentos revisados, el $56 \%$ corresponde a publicaciones desde los propios movimientos, como resultados de ejercicios reflexivos y de investigaciones.

Respecto a los autores, 72 provienen de las ciencias sociales, principalmente, de la sociología y la antropología; la presencia de profesionales de la educación y la pedagogía ha venido acentuándose en las dos últimas décadas, en particular de aquellos que se asumen como educadores populares; la mayoría forma parte de colectivos académicos que acompañan procesos sociales.

Los países de mayor producción en el campo son Argentina (25 registros), Colombia (23) y Brasil (19); en estos hay investigadores, como la brasileña Roselli S. Caldart, y grupos de investigación con sostenida producción, como la Cooperativa de educadores e investigadores sociales de la Universidad de Buenos Aires (Marina Ampudia y Roberto Elisalde), y el grupo "Sujetos y nuevas narrativas en investigación en ciencias sociales", de la Universidad Pedagógica Nacional de Colombia.

En Brasil, la educación en movimientos campesinos, especialmente del Movimiento de los Trabajadores Rurales Sin Tierra (MST), es el caso más analizado desde sus propuestas políticas y pedagógicas tanto por investigadores consagrados como Roselli Saleti Caldart, Norma Michi, Oscar Ospina y Fabián Regalado, como por numerosos tesistas. En Argentina, atraen interés, la Universidad de las 
Madres de la Plaza de Mayo, primero, y luego los bachilleratos populares en la ciudad y en la provincia de Buenos Aires. En México, las escuelas zapatistas son las que más atraen la atención de los investigadores, los cuales elaboran sus planteamientos desde la educación indígena, el género y la política.

En términos metodológicos, algunos son estudios comparativos de varios casos de movimientos en uno o varios países; otras son investigaciones cualitativas que describen y analizan una propuesta específica; tienden a crecer las investigaciones con algún carácter participativo y las sistematizaciones de experiencias.

En cuanto a su despliegue temporal, en la década de 1980, los primeros estudios los encontramos referidos a la educación en experiencias organizativas, a las cuales se les interroga desde la política, sea para evaluar su potencial transformador (Palma, 1986) o para valorar sus aportes a la construcción de democracia (Bengoa, 1987). El trabajo de Caldart (1988) es pionero en explorar los vínculos entre los Ms y la producción de subjetividades, en particular en la afectación de marcos cognitivos y valorativos.

A lo largo de la década de 1990, con el paulatino desmonte de las dictaduras militares, la crisis del socialismo histórico del bloque soviético, los procesos de paz llevados a cabo en Guatemala, El Salvador y Colombia, y principalmente con la generalización de la implantación del modelo económico neoliberal, hubo una reactivación de las luchas y Ms, y con ella, del interés por estudiarlos; en nuestro caso, se reactiva tímidamente el interés por indagar el lugar de lo educativo en dichas dinámicas de acción colectiva.

Como han planteado varios autores, desde la década de 1990 los ms complejizan sus demandas ya no solo centradas en la clase sino desde la configuración de identidades, luchas territoriales y demandas desde otros campos como el ambiental, debido a la explotación minera. Aquí encontramos las producciones de Brasil en torno a lo educativo en los nuevos MS (Gohn, 1992) y en Colombia con las organizaciones populares urbanas (Torres, 1995). En ambos casos, el resultado de las investigaciones es desde dentro de las organizaciones y como reflexión de las prácticas educativas y su aporte al proyecto político.

En los años 2000, continúan los tratados de libre comercio (TLC) y la tercerización laboral a través de cooperativas. Hay un repunte de los movimientos de género, el feminismo y grupos LGTBI. Además, el ascenso de gobiernos sociales como Argentina y Chile y otros más populares como el de Ecuador y Bolivia. En Chile, Los Pingüinos acompañados de sus maestros, paralizan el país en búsqueda de un cambio educativo; y en Argentina, con la fuerte crisis del Corralito, se recuperan fábricas. En este periodo se identifican temáticas generales como Ms y América Latina (Garcés, 2003, Zibechi, 2003); subjetividades y sujetos en la educación (Gómez, 2001; Torres, 2000, 2006, 2009); la educación y los Ms (Ampudia y Elisalde, 2008); MS, educación popular y educación para personas jóvenes y adultas (EPJA) (Ruíz, 2005; Goldar, 2008, Freire, 2005; Mafra et al., 2008).

Temáticas más específicas y situadas en las organizaciones y Ms plantean: a) la organización como escenario educativo: Universidad Popular Madres Plaza de Mayo (Basile, 2002), las organizaciones populares urbanas (Barragán, Mendoza y Torres, 2006); b) la educación en sujetos sociales específicos como MST (Caldart, 2000, 2003, 2004); los jóvenes y adultos (Ampudia, 2007); bachilleratos populares en empresas recuperadas (Ampudia y Elizalde, 2008, 2008; Sverdlick y Costas, 2008); feminismo (Ochoa, 2008; Padierna, 2009); y c) pedagogías de los Ms: de la tierra (Ferrari, 2003); educación para la solidaridad (Araus, 2011), pedagogía del movimiento (Caldart, 2000, 2003, 2004; Madeiros, 2010; Michi, 2010).

Entre 2011 y 2017 continúan las tendencias anteriores, junto con la emergencia de balances reflexivos sobre la educación en los Ms. En el primer caso, están los estudios y reflexiones sobre movimientos emblemáticos como el EZLN (Padierna, 2012), los bachilleratos populares (Ampudia y Elizalde, 2014); los movimientos campesinos y el MST (Araus, 2011); las organizaciones populares (Aguilera, González y Torres, 2015).

En cuanto a los balances reflexivos y críticos de la producción sobre el tema, tenemos: estados del arte 
sobre educación en movimientos argentinos y brasileños (Baraldo, 2010; Palumbo, 2016); valoraciones de las luchas populares por los derechos humanos o el derecho a la educación (Mardonés, 2012; Gluz, 2013); la educación y la acción colectiva (Delgado, 2011, 2012); preguntas por la sostenibilidad de las propuestas (Aguilera y González, 2014); las posibilidades y desafíos de la interculturalidad (Gashé, 2010; González, 2013; Baronnet, 2012, 2013; Alban, 2015); aportes desde y hacia la educación popular (Bustos et al., 2012; Korol, 2016; Torres, 2016).

A partir del análisis transversal del contenido de todos los documentos, se identifican cinco temas recurrentes en esta trayectoria de tres décadas de estudios sobre educación en MS: las diferentes comprensiones de lo educativo en los movimientos; la formación de militantes como sentido de las propuestas; la organización o el movimiento como sujeto educativo; las pedagogías de los movimientos como aporte epistémico, pedagógico y político, y la formación de subjetividades y sujetos sociales. A continuación, nos ocuparemos de cada uno de ellos.

\section{¿Qué es lo educativo en los movimientos sociales?}

Una primera consideración de los estudios acerca de lo educativo en organizaciones y movimientos sociales es reconocer que destacan su posicionamiento crítico y su potencialidad para transgredir la educación institucionalizada y reivindicar otros sentidos educativos, llámense liberadores, emancipadores o alternativos. En la medida en que se articulan y se subordinan a los sentidos políticos de las organizaciones y acciones colectivas en las que surgen, también se proponen transformar las relaciones injustas de poder y saber, promover el diálogo de saberes, la inclusión de los contenidos de la vida cotidiana y la autonomía de los sujetos individuales y colectivos (Caldart, 2003; Torres, 2006; Ochoa, 2008; Padierna, 2010; ENEOB, 2010; Baronnet, 2010; Aguilera, González y Torres, 2015; Pinheiro, 2016; Korol, 2016; Barragán y Torres, 2017; Torres, 2017).

Junto a esta comprensión de la educación agenciada desde los movimientos y organizaciones como una práctica crítica y transformadora a la hegemónica, en las investigaciones la educación es comprendida como un proceso en el que los sujetos se dan forma y al hacerlo buscan transformar el mundo; el propósito de la educación es formativo y busca constituir sujetos históricos de cambio en donde lo específicamente educativo es la intención de afectar la conciencia, los saberes y los modos de hacer (Barragán, Mendoza y Torres, 2006; Ochoa, 2008; Aguilera, González y Torres, 2015).

De esta manera, la educación en y desde la acción colectiva es una práctica que desnaturaliza las ideas de una realidad fija y determinada, y favorece la criticidad y capacidad de acciones emancipatorias de los sujetos; quienes al reconocer que las instituciones no son dadas sino construidas socialmente, se oponen a un destino dictado por leyes y orientaciones deterministas.

Así que lo formativo es crítico en la medida en que aporta a la configuración de subjetividades con identidades, prácticas y orientaciones para la acción transformadora. Esta formación produce un tipo de identidad, un nosotros que afecta diversas maneras de ser, hacer y comprender el mundo, en las que el autoconcepto y el sentido de pertenencia se amplían hacia grupos e intereses emancipatorios (Torres, 2000; Zibechi, 2003; Barragán, Mendoza y Torres, 2003; Ruíz, 2005; Guelman, 2010; Pascual, 2012).

Para estos autores, la educación y la formación en tanto procesos se relacionan con la construcción de sentidos y de experiencia en las personas, que surgen de la reflexión de las prácticas o modos de hacer educativos y que son posibles de construir en tanto afectan los significados que los sujetos portan sobre los asuntos que les conciernen y marcan sus tiempos, actividades cotidianas y proyecto colectivo. En este caso, los procesos formativos pueden reflexionarse desde su capacidad instituyente y creativa para actualizar las demandas del movimiento.

En consecuencia, algunos estudios evidencian que todo lo educativo, por cuanto práctica social, no es emancipatorio; lo es cuando logra en sus fines lo formativo, es decir, cuando la práctica educativa se constituye en experiencia, y permite a los colectivos 
sociales construir nuevos sentidos sobre los sujetos y objetos de conocimiento, particularmente sobre el mundo social (Freire, 2005; Barragán, Mendoza y Torres, 2006; Michi, 2010; Svampa, 2010; Araus, 2011; Pascual, 2012; Aguilera, González y Torres, 2015).

Finalmente, para algunos autores, a partir de lo planteado por los propios movimientos y sus propuestas educativas, la educación es asumida como un derecho social, cuya atención es responsabilidad del Estado. En particular, las escuelas o procesos formativos de movimientos como MST y bachilleratos populares se enmarcan en torno a este planteamiento de la educación como derecho (Araus, 2011; Guelman, 2010; Gluz, 2013; Mardones, 2012; Sverdlick, 2008).

\section{La formación de militantes, apuesta educativa de los MS}

El reconocimiento de la relación entre demandas de los movimientos sociales y crisis del modelo neoliberal es comprendido como un proceso tensionado por la lucha social y su continua capacidad creativa y de resistencia, que lee y actualiza repertorios y exigencias desde su contacto con los sectores subalternizados y empobrecidos.

La fuerza emancipatoria de lo educativo se produce al fortalecer en los sujetos capacidades de lectura de contexto y análisis de críticas (orientadas a la transformación), que favorecen la afirmación de propuestas de vida digna alternativas al modelo económico actual y que se expresan en las diversas propuestas pedagógicas e interpelan las relaciones verticales de saber y poder de la educación convencional (Aguilera, González y Torres, 2015; Barragán, Mendoza y Torres, 2003, 2006; Gómez, 2001; Ochoa, 2008; Padierna, 2010; Ruíz, 2005; Caldart, 2003; Torres, 2000).

Un acuerdo entre los autores es la consideración de que en la búsqueda de transformación de la sociedad injusta, los movimientos sociales y organizaciones, comprenden lo educativo y los procesos formativos que desarrollan, como estrategia de configuración de subjetividades con al menos tres propósitos: a) la formación de seres humanos justos y solidarios; b) la conformación de identidades colectivas e individuales que se reconocen en la acción social y resistentes al modelo hegemónico, y c) la construcción de sentidos y prácticas educativas y políticas para la construcción de proyectos alternativos.

Algunos autores consideran que los procesos formativos en las organizaciones son instituyentes y desafían el orden hegemónico a través de proponer otros mundos posibles, afirmar la diferencia, lo colectivo y la solidaridad; en fin, al plantear a los sujetos como productores y socializadores de sentidos e identidades que promueven acciones políticas, económicas, sociales y culturales alternativas (Lamus, 2006; Michi, 2010; Aguilera, González y Torres, 2015). Así, favorecen que los sujetos sociales desnaturalicen imaginarios que los subordinan y propongan alternativas epistémicas (Albán, 2015) que los afirman como sujetos con saberes y experiencias trasformadoras e instituyentes.

La categoría diferencia es propuesta por algunos autores para expresar las demandas de los movimientos y organizaciones sociales desde la reivindicación de otras dimensiones identitarias como el territorio, la etnia o los géneros (Zibechi, 2003; Torres, 2006; ENEOB, 2010), y que encuentran en los procesos educativos modos de hacer que interpelan a los y las participantes al relacionarse con núcleos o rupturas identitarias.

Esta función identitaria se fortalece con la visibilización de las trayectorias de los sujetos subordinados y de sus experiencias en pro del cambio social, de manera que se reconoce su protagonismo y capacidad de incidencia en la vida cotidiana. Esto quiere decir que la comprensión de los sentidos de pertenencia incluye las percepciones, representaciones, autoimágenes que los individuos, tanto los portadores de esa identidad como los que la reconocen y definen, tengan acerca de lo identitario en el proceso educativo (Barragán, Mendoza y Torres, 2006; Brito, 2008; Castells, 2017).

\section{La organización como lugar educativo}

El planteamiento de la educación alternativa a la bancaria, al interior de los Ms busca la visibilización 
y condiciones de igualdad de los sectores populares, como sujeto histórico (Burgos, 2014). Uno de los principales aportes de las experiencias formativas de los movimientos sociales es interpelar a la escuela como escenario privilegiado para la formación (Gluz, 2013; Guelman, 2010; Michi, 2010; Padierna, 2009; Palumbo, 2016; Caldart, 2003), al autocomprenderse como sujeto y escenario educativo que materializa la transformación de relaciones de poder y saber entre educadores y educandos.

Al considerar que todo es educativo (Barragán, Mendoza y Torres, 2006) es posible que las actividades de la organización tengan altos grados de significación para las personas debido a su cercanía con el contexto particular, su problematización de lo cotidiano, su desnaturalización de prejuicios, sus posibilidades pragmáticas de reorientación de las comprensiones sobre el mundo de la vida, etc.; de manera que las organizaciones se constituyen en escenarios educativos al dotar a los sujetos con nuevas capacidades y herramientas para comprender y transformar la realidad (Caldart, 2003; Palumbo, 2016).

La organización como escenario educativo rompe con la idea de tiempos determinados para formarse y proponerla a lo largo de la vida (en concordancia con las discusiones de la educación para jóvenes y adultos), problematizando las representaciones de edades y tiempos para aprender, que desconocen y marginan a los adultos mayores como sujetos productores de saber y experiencia. En múltiples investigaciones se plantea el espacio de la fábrica, el sindicato, las reuniones de las organizaciones y el acompañamiento que hacen los fundadores como espacios formativos (Barragán, Mendoza y Torres, 2003; Caldart, 2003; Ruíz, 2005; Zanguña, 2017).

Esta condición de escenario formativo de las organizaciones y movimientos sociales, interpela relaciones de poder y saber entre los activistas, al producir múltiples interacciones mediadas por los sentidos del proyecto colectivo; y confirma la posibilidad de construcción de saberes y conocimientos desde los activistas y su experiencia en las organizaciones; es decir, abre paso a la elaboración de epistemologías propias que promueven modos de pensar y hacer situados (Albán, 2015; Barragán y Torres, 2017; Gasché, 2010; Pinheiro, 2016; Torres, 2017).

\section{Pedagogías en movimiento}

Autores como Gohn (1992), Caldart (2000), Zibechi (2005), Bidaseca (2006), Korol (2007) y Michi (2010) señalan que los procesos formativos son un eje esencial en la consolidación de los movimientos sociales, destacando el componente pedagógico de los procesos sociales y políticos de los movimientos, en lo referente a la creación de nuevos valores, sentidos y representaciones.

La pedagogía de Paulo Freire y la educación popular son mencionadas como referentes de las propuestas educativas analizadas; en particular, su reconocimiento del carácter político de la educación, sus intenciones emancipadoras, los sentidos de transformación social y sus planteamientos de esperanza e inédito viable, así como el reconocimiento del saber de los sujetos y su capacidad emancipadora, el uso de metodologías dialógicas y participativas, como estrategias que vinculan la educación y la acción política (Barragán, Mendoza y Torres, 2006; Michi, 2010; Ochoa, 2008; Araus, 2011; Pascual, 2012; Gluz, 2013; Pinheiro, 2016; Torres, 2017; Zanguña, 2017).

La manera específica como se disponen los contenidos, sus fines y resultados esperados se ha denominado currículo explícito para diferenciarlo de su puesta en acción que dependerá de los saberes y postura de los agentes educativos frente a las orientaciones planteadas. Es en esta medida que el currículo es pensado como espacio de disputa de los colectivos populares por incluir sus intereses, comprensiones y experiencias (Gasché, 2008; González Arroyo, 2010).

Plantean los autores estudiados que la selección cultural de los contenidos formativos es una acción de resistencia epistémica desde la afirmación de lo educativo como acción política que atiende a su expresión en el territorio, a los tiempos y espacios en que las comunidades los realizan y que posibilitarán que se convierta en un modo de hacer (Gasché, 2008; Sverdlick y Costas, 2008; Da Silva, 2012), que aportará a la realización del proyecto colectivo alternativo. 
Además, reconocer estos saberes en las actividades de las comunidades permite que los formadores (muchas veces participativamente) realicen diseños curriculares pertinentes, al comprender el sentido político y social de los contenidos seleccionados en cada comunidad y proponer un diálogo intercultural crítico con las propuestas formales desde el respeto a la diferencia y la complementariedad epistémica (Caldart, 2013; Gasché, 2008, 2010; Da Silva, 2012; Gluz, 2013; Barragán y Torres, 2017; Torres, 2017).

En cuanto construcciones históricas, los procesos formativos presentan altos grados de complejidad e incertidumbre, que encuentran respuestas en las capacidades expresadas por su militancia y la actualización de su proyecto colectivo.

\section{Producción de subjetividades emancipadoras en los procesos educativos de los movimientos sociales}

La temática de la constitución de subjetividades sociales es un legado de Hugo Zemelman a la discusión latinoamericana; la categoría de sujeto como un interrogante político es luego asumida como ángulo de construcción epistémica, desafío pedagógico e imperativo ético. Nace de la crisis y desafíos identificados por teóricos y militantes, a partir del golpe militar en Chile y el suicidio de Allende en 1973. Exigencia que lleva a Zemelman $(1989,1992)$ a plantear a los sujetos sociales como ángulo de conocimiento y lente metodológico.

En la misma línea y desde organizaciones populares se encuentran trabajos en Colombia, fundamentados en la educación popular (Torres, 1993, 1994; Cuevas, Naranjo y Torres, 1996). Campo investigativo que desde perspectivas interpretativas se formaliza en el grupo de investigación de la UPN "Sujetos y nuevas narrativas en investigación y enseñanza de las ciencias sociales" (Torres y Torres, 2000; Torres, 2000).

En las elaboraciones teóricas los autores la comprenden como una construcción intersubjetiva de sentidos en la que confluyen procesos estructurantes del orden social, sin determinarla; así como dinámicas no estructuradas que posibilitan la emergencia de sentidos instituyentes que les devuelven a los sujetos su capacidad de agencia; así mismo, la subjetividad es asumida no solo como una temática social, sino como un lente de análisis de la realidad social (Contreras y Nuria, 2010; Torres, 2000, 2006, 2010; Zemelman, 1997, 2002).

En cuanto productora de sentido, Torres (2006) plantea unas funciones sociales de la subjetividad: aportar marcos interpretativos de la realidad, la de orientar la acción individual y colectiva, y la de significar vínculos y claves de identificación y pertenencia social. De esta manera, la participación en movimientos y organizaciones sociales amplía sus marcos de interpretación/construcción de significados, potencia su capacidad de transformación y construcción de realidades, y afirma o produce identidades estratégicas.

Los estudios sobre la relación entre ms y educación coinciden en señalar sentidos y posibilidades instituyentes y producción de subjetividades emancipadoras; además, alertan sobre desafíos en la relación con la institucionalidad, sobre su coherencia interna con el proyecto colectivo y su sostenibilidad, y la ampliación del diálogo de la pedagogía de los sujetos sociales y subjetividades emancipadoras con otros campos sociales críticos (Aguilera, González y Torres, 2015).

\section{Desafíos a las propuestas formativas de los movimientos sociales}

Los desarrollos y desafíos de las propuestas educativas obedecen a prácticas instituyentes en permanente tensión por la exigencia de su pertinencia política y social del lado de la militancia, y a su articulación con sistemas formales, desde el lugar institucional (Aguilera y González, 2014; Baronnet, s.f.; Caldart, 2003; Svampa, 2010; Sverdlick y Costas, 2008; Torres, 2017).

Así que un primer desafío es que efectivamente el movimiento social comprenda y profundice lo formativo en todas sus actividades, como expresión de su capacidad de agencia y escenario pedagógico en búsqueda permanente de apuestas más democráticas y participativas. 
En el caso de las escuelas de formación que además de la formación de militancia y elaboración de proyectos alternativos, demandan la educación como derecho y las responsabilidades del Estado en su continuidad, sostenibilidad y la certificación de los estudiantes; el desafío refiere a la capacidad de diálogo entre la autonomía del movimiento y su resistencia a la instrumentalización, y su reconocimiento institucional.

Relacionado con el anterior, el reto refiere a la sistematización de los modos de hacer pedagógicos que resulten instituyentes y promuevan sujetos emancipadores; de manera que se realice un continuo diálogo entre el hacer, el pensar y el actuar.

Un tercer desafío es reconocer las diferentes prácticas que vienen configurando un campo pedagógico instituyente para establecer puentes explícitos entre la academia y las organizaciones sociales que desconcentre el discurso de los grupos de investigación e ingrese en los currículos de formación profesional. Esta apertura enriquecerá sin duda la praxis de los MS que, sirviéndose de la responsabilidad política de la academia, pueda conectarla con los intereses y experiencias desde los bordes y desde abajo.

\section{Conclusiones}

La relación entre educación y Ms ha sido un tema constante en los estudios, que iniciado desde teorizaciones generales se ha venido enraizando en las prácticas singulares de actores sociales específicos.

El carácter magmático de la lucha social ha exigido a los investigadores militantes esfuerzos conceptuales y metodológicos para su comprensión y teorización; a la vez, ha obligado a ampliar los marcos de interpretación de las disciplinas sociales.

El tema de las subjetividades emancipadoras es relativamente nuevo, pero se ha venido incrementando desde la última década del siglo pasado. A pesar de ser una preocupación en América Latina, es una inquietud diferencial para núcleos académicos colombianos.

La revisión de los estudios muestra la importancia de seguir ampliando los escenarios de reflexión entre académicos y movimientos sociales para reconocer y superar desafíos epistémicos, políticos e investigativos.

El estudio muestra una debilidad de la pedagogía como disciplina en el abordaje de la relación entre educación y movimientos sociales que, por su importancia en la sistematización y reflexividad de las experiencias sociales, exige mayor continuidad y profundidad.

\section{Referencias}

Aguilera, A. y González, I. (2014). Educación y movimientos sociales. La sostenibilidad de las propuestas. Folios, 39, 117-135.

Aguilera, A., González, I. y Torres, A. (2015). Reinventando la política. Formación de subjetividades, sentidos de comunidad y alternativas políticas en procesos organizativos locales. Bogotá: UPN.

Albán, A. (15 de julio de 2015). Educación e interculturalidad en sociedades complejas. Tensiones y alternativas. Recuperado de goo.gl/Pii5wX

Ampudia, M. (2007). El sujeto de la educación para jóvenes y adultos. Territorialización y desterritorialización de la periferia. Buenos Aires: CEIP.

Ampudia, M. (2012). Movimientos sociales y Educación Popular. Reflexiones sobre la experiencia educativa de los Bachilleratos Populares. Revista osera, 6.

Ampudia M. y Elizalde, R. (2008). Movimientos sociales y educación. Buenos Aires: Buenos Libros.

Ampudia M. y Elisalde, R. (2014). Los bachilleratos populares en la Argentina: Organizaciones sociales y movimiento pedagógico. Educación y Cultura, $105,28-33$.

Apple, M. (1987). Educación y poder. Barcelona: Paidós.

Araus, M. (2011). El movimiento de los sin tierra y su planteamiento educativo (primera parte). Educación para la solidaridad. Recuperado de http://educacionysolidaridad.blogspot.com/2011/02/el-movimientode-los-sin-tierra-y-su.html

Araus, M. (2011). El movimiento de los sin tierra y su planteamiento educativo (segunda parte). Recuperado de http://educacionysolidaridad.blogspot.com/2011/02/ el-movimiento-de-los-sin-tierra-y-su_17.html

Baraldo, N. (2010). Educación en y desde los movimientos sociales: ¿nuevo objeto y nuevos abordajes en Educación? Cuadernos de Educación, 8, 23-39. 
Baronnet, B. (2012). Autonomía y educación indígena. Las escuelas zapatistas de la Selva Lacandona de Chiapas. Quito: Abya-Yala.

Baronnet, B. (2013). Movimientos sociales y educación indígena en América Latina. México: UnAM.

Barragán, D., Mendoza, C. y Torres, A. (2003). Organizaciones populares, identidades colectvas $y$ ciudadanía en Bogotá. Bogotá: UPN.

Barragán, D., Mendoza, C. y Torres, A. (2006). Aquí todo es educativo. Folios, 23,15-28.

Basile, M.T. (2002). La Universidad Popular de las Madres de Plaza de Mayo: emergencia de nuevas prácticas en cultura y poder en la Argentina de la Posdictadura. Estudios y otras prácticas intelectuales latinoamericanas en cultura y poder. Buenos Aires: Clacso.

Batista, M. S. (2010). Movimentos sociais e educação popular do campo. Reconstituindo território e identidade camponesa. En E. Jezine y M.L. de Almeida (eds.), Educação e movimentos sociais (pp. 169-190). Campinas: Alínea.

Bengoa, J. (1987). La educación para los movimientos sociales. Proposiciones, 15. Chile.

Burgos, A. (2014). Política y educación: experiencias educativas alternativas en movimientos sociales. Buenos Aires: Universidad de La Plata, Facultad de Periodismo y Comunicación Social.

Bustos, L. Oyarzún, E., Órdenes, D., Acevedo, C., Santander, P., Lara, E., Fredericksen, J., Silva, J. y Fauré, D. (2012). Somos andando. Prácticas, caminos y saberes para construir educación popular hoy. Santiago: Editorial Quimantú.

Caldart, R.S. (1988). Pedagogía de los movimientos sociales. En J. Osorio y L. Weinstein. La Fuerza dei Arco iris (pp. 123-145). Santiago: CEAAL.

Caldart, R.S. (2000). O MST e a formação dos Sem Terra: o movimento social como principio educativo en: la ciudadanía negada. Políticas de exclusión en la educación y el trabajo. Buenos Aires: Clacso.

Caldart, R.S. (2003). Movimento Sem Terra: lições de Pedagogia. Currículo sem Fronteiras, 3(1), 50-59.

Caldart, R.S. (2004). Pedagogia do movimento sem terra. São Paulo: Expressão Popular.

Carr W, S. K. (1988). Teoría crítica de la enseñanza. Investigación acción en la formación del profesorado. Barcelona: Martínez Roca.

Castells, M. (21 de marzo de 2017). Globalización, identidad y Estado en América Latina. Recuperado de http://red.pucp.edu.pe/wp-content/uploads/ biblioteca/081219.pdf: http://red.pucp.edu.pe/wpcontent/uploads/biblioteca/081219.pdf

Contreras, J., y Pérez, N. (2010). La experiencia y la investigación educativa. En N. Contreras y N. Pérez (coord..), Investigar la experiencia educativa (pp. 21-86). Madrid: Morata.

Cuevas, P., Naranjo, J. y Torres, A. (1996). Discursos, prácticas y actores de la educación popular en Colombia. Bogotá: Universidad Pedagógica Nacional

Da Silva, L.J. (2012). El método inductivo intercultural y el Calendario Socioecológico como estrategias para el fortalecimiento de una formación crítica e intercultural de profesores indígenas de los estados de Minas Gerais y Bahía, Brasil. Revista ISEES, 10, 79-94,

De Certeau, M. (1996). La invención de lo cotidiano. I. Artes de hacer. México D.F.: Universidad Iberoamericana.

Delgado, R. (2011). Acción colectiva y educación popular: contribuciones para un conocimiento emancipatorio. Folios, 33, 53-61. Bogotá: UPN.

Delgado, R. (2012). Educación para la ciudadanía desde la acción colectiva. Magis, 4(7) 201-210.

Delgado, R., Martínez, M.C. y Torres, A. (2012). Seminario temático: experiencias educativas y pedagógicas en los movimientos sociales de América Latina, UPN, Bogotá, Doctorado Interinstitucional en Educación, DIE.

De Rugeriis, R. (2010). Reseña Comunicación, Educación y Movimientos Sociales en América Latina de César Bolaño, Sônia Meire de Jesus y Verlane Aragão Santos. Quórum Académico,(7),2, 156-161.

Dietz, G. (2012). Multiculturalismo, interculturalidad y diversidad en educación. Una aproximación antropológica. México D.F.: Fondo de Cultura Económica.

ENEOB (2010). Experiencias educativas contrahegemónicas. La pedagogía del oprimido como formación política para la organización. En E.N. Base, Primer foro de educación para el cambio social (pp. 239-265). Buenos Aires: El Colectivo.

Ferrari, S. (2003). El Movimiento Sin Tierra y sus propuestas de futuro. "Actores de una pedagogía de la tierra”. Una entrevista de Sergio Ferrari a Marcia Mora Ramos, dirigente nacional del área educación del Movimiento de los Sin Tierra (MST). Recuperado de https://www.prensarural.org/brasil20031019.htm 
Fragoso, M. (2000). Pedagogia do Movimento Sem Terra: escola é mais do que escola. Rev. Bras. Educ., 15, 177-179.Recuperado de http://www.scielo.br/ scielo.php?script $=$ sci_arttext $\&$ pid $=$ S1413-24 782000000300011\&lng=en\&nrm=iso

Freire, P. (2005). Pedagogía del oprimido. Buenos Aires: Siglo XXI.

Garcés, M. (2003). Los movimientos sociales en América Latina en el actual contexto. Córdoba: ETS-UNC.

Gasché, J. (2010). ¿Qué son “saberes" o “conocimientos" indígenas, y qué hay que entender por "diálogo"? En Memorias del 1 Encuentro Amazónico de experiencias de Diálogo de saberes. Leticia: Universidad Nacional de Colombia, sede Amazonía.

Gasché, J. (2010). De hablar de la educación intercultural a hacerla. Mundo Amazonico, 1, 111-134.

Gimeno, J. (2010). ¿Qué significa el curriculum? En J. Gimeno, Saberes e incertidumbres sobre el curriculum (pp. 19-43). Madrid: Morata.

Gimeno, J. (2015). Los contenidos. Una reflexión necesaria. Madrid: Morata.

Gluz, N. (2013). Las luchas populares por el derecho a la educación: experiencias educativas de movimientos sociales. Buenos Aires: Clacso.

Gohn, M.G. (1992). Movimentos sociais e educação. $2 \mathrm{a}$. ed. San Pablo: Cortez.

Gohn, M.G. (2010). Movimentos sociais e redes de mobilizações civis no Brasil contemporâneo. Petrópolis: Vozes.

Goldar, M.R. (2008). Los movimientos sociales y los desafíos de la EP. Apuntes para una reflexión al interior del CEAAL. La Piragua, 29, 34-45.

Gómez, M. (2001). Formación de sujetos de la educación y configuraciones epistémico - pedagógicas. En B.O. Gómez Marcela y B. Orozco (comp.), Pensar lo educativo. Tejidos conceptuales (pp. 51-73). México D.F.: Plaza y Valdés Editores.

González, M. (2010). Los colectivos depauperados repolitizan los currícula. En J. Gimeno Sacristán (comp.), Saberes e incertidumbres sobre el curriculum (pp. 128-148). Madrid: Morata.

González, M.I. (2011). Movimiento indígena y educación intercultural en Ecuador, México, UNAM-Clacso.

González, M.I. (2013). Las convergencias educativas de movimientos sociales en América Latina: transitando otros caminos. Ideação, 15(1), 46-62.
González, M.I. (2013). Las convergencias educativas de movimientos sociales en América Latina: transitando otros caminos. Ideação, 15(1).

González, M.I. (2014). Organización indígena y educación. Un binomio indisoluble en Ecuador. Educación Populary Cultura, 105.

Granja, J. (2001). Análisis conceptual del discurso. Un instrumento para observar la producción de conocimientos en el campo de la educación. En B. O. Gómez Marcela, Pensar lo educativo. Tejidos conceptuales (pp. 17-26). México, D.F: Plaza y Valdés Editores.

Guelman, A. (2010). Movimientos Sociales y Educación. Tesis 11. Recuperado de http://www.tesis11.org.ar

Korol, C. (2004). Pedagogía de la resistencia. Ediciones Madres de la Plaza de mayo, Buenos Aires.

Korol, C. (2007). La formación política de los movimientos populares latinoamericanos. OSAL, VIII, 22.

Korol, C. (comp.) (2016). Pedagogía de las revoluciones. Buenos Aires: Ediciones América Libre. En Mafra, J., Gómez, M.V., Gadotti, M., y Fernandes de Alentar, A. (comp.) (2008). Paulo Freire. Contribuciones para la pedagogía. Buenos Aires: Clacso.

Madeiros, L.B. (2010). El mst y la lucha emancipadora desde la educación en el campo en Brasil. Alternativas, 39, 87-104.

Mardonés, R. (2012). Movimientos sociales, derechos humanos y educación: hacia una visión compleja desde el pensamiento de Emmanuel Levinás. Revista Electrónica de Psicología Política, 27, 96-111.

Mejía, Marco R. (1996) Educación popular y movimientos sociales.

Michi, N. (2010.). Movimientos campesinos y educación. El movimiento de los trabajadores rurales sin tierra y el Movimiento campesino de Santiago del Estero - VC. Buenos Aires: El Colectivo.

Mignolo, W. (2003). Historias locales / diseños globales: Colonialidad, conocimientos subalternos y pensamiento fronterizo. Editorial akal, Madrid, España.

Ochoa, L.M. (2008). El sueño y la práctica de sí. Pedagogía feminista. Una propuesta. México, D.F.: Colegio de México.

Padierna, M. (2010). Educación y movimientos sociales. Pampedia, 6, 13-27.

Palma, D. (1986). Movimientos sociales y educación necesaria. Revista Acción Crítica, 19, 45-56. Lima, Perú. 
Palumbo, M.M. (2016). Educación en movimientos populares rurales: un estado del arte. Historia de la Educación, 18(26), 219-240.

Pascual, M. (2012). Movimientos populares y educación en América Latina. Revista El Ecologista, 73. Recuperado de https://www.ecologistasenaccion. org/article10496.html

Pinheiro, L. (2016). Educación, resistencia y conocimiento en América Latina: por una teoría desde los movimientos sociales. De Raíz Diversa, 3(6), 45-79. Universidade Estadual Do Ceará.

Rockwell, E. (2012). Movimientos sociales emergentes y nuevas maneras de educar. Educación y Sociedad, Universidad de Campiñas, 33(120), 697-713.

Ruíz, M. (2005). Imbricación de lo político y lo pedagógico en los procesos de educación de adultos: Dos estudios en caso. México: Centro de Cooperación Regional para la Educación de Adultos en América Latina y el Caribe Pátzcuaro.

Souza, M.A. (2007). A pesquisa sobre educação e o Movimento dos Trabalhadores Rurais Sem Terra (MST) nos Programas de Pós-Graduação em Educação. Revista Brasileira de Educação, 12(36), 443-548.

Svampa, M. (2010). Apertura. En Primer Foro Nacional de Educación para el Cambio Social (pp. 7-20). Buenos Aires: El Colectivo.

Sverdlick, I. y Costas, P. (2008). Bachilleratos populares en empresas recuperadas y organizaciones sociales. Ensayos \& Investigaciones del Laboratorio de Políticas Públicas - Buenos Aires, № 30.

Sverdlick, I. y Costas, P. (2010) Apertura en encuentro nacional de estudiantes de organizaciones de base. ENEOB, Argentina.

Tapia, G. (1987) La producción de conocimientos en el medio campesino. PIIE, Chile.

Torres C., A. (1993). La educación popular y la pedagogía. Evolución reciente y actuales búsquedas. La Piragua, 7, 24-31.

Torres C., A. (1995) Educación y pedagogía en las experiencias organizativas populares. La Piragua, $11,49-54$.

Torres C., A. (2000). Sujetos y subjetividad en la educación. Educación popular, subjetividad y sujetos sociales. Pedagogía y Saberes, 15, 5-14.
Torres C., A. (2006). Subjetividad y sujeto. Perspectivas para abordar lo social y lo educativo. Bogotá: UPN.

Torres C., A. (2009) Acción colectiva y subjetividad. Un balance desde los movimientos Sociales". Folios 30, 51-74.

Torres C., A. (2011). Movimientos sociales. Trayectorias históricas y desafíos contemporáneos. Bogotá: Grupo editorial Educar.

Torres C., A. (2017). Prácticas educativas en movimientos sociales de América Latina. Folios, 46, 3-14.

Torres, A. y Torres, J.C. (2000). Sujeto y subjetividad en la obra de Hugo Zemelman. Folios, 12, 12-23.

Torres, J. (1994). Globalización e interdisciplinariedad: el curriculum integrado. Madrid: Morata.

Torres, J. (2012). La justicia curricular. El caballo de Troya de la cultura escolar. Madrid: Morata.

Triana, D. (2012). Autonomía y autogestión, un horizonte político pedagógico. Los bachilleratos populares en Buenos Aires 2001-2012 (tesis inédita de maestría). Universidad Pedagógica Nacional, Bogotá, Colombia.

Walsh, C. (2009). Interculturalidad y (de)colonialidad. Perspectivas críticas y políticas. Ponencia preparada para el XII Congreso ARIC. Florianópolis, Brasil.

Walsh, C. (2014). Lo pedagógico y lo decolonial: Entretejiendo caminos. Querétaro, Mexico.

Zanguña, R. (2017). Las transformaciones subjetivas de los $y$ las jóvenes pertenecientes a los procesos formativos comunitarios de a organización social de base ASOVEGCINJUDESCO durante el 2000 al 2015 en la localidad de Rafael Uribe Uribe. Bogotá: Universidad Distrital Francisco José de Caldas, Maestría en Investigación Social Interdisciplinar.

Zemelman, H. (1989). De la historia a la política. México: Siglo XXI.

Zemelman, H. (1992). La educación en la construcción de sujetos sociales. La Piragua, 7, 12-18.

Zibechi, R. (2003). Los movimientos sociales latinoamericanos. Tendencias y desafíos. Observatorio Social de América Latina, OSAL, 9, 185-188.

Zibechi, R. (2005). Educación en movimientos sociales. El Programa de las Américas del IRC. Ircaméricas.org

\section{Para citar este artículo}

Barragán, D. y Torres, A. (2018). Estudios sobre procesos educativos en organizaciones y movimientos sociales. Folios, 48, 15-25. 